\title{
GMDH: An R Package for Short Term Forecasting via GMDH-Type Neural Network Algorithms
}

by Osman Dag and Ceylan Yozgatligil

\begin{abstract}
Group Method of Data Handling (GMDH)-type neural network algorithms are the heuristic self organization method for the modelling of complex systems. GMDH algorithms are utilized for a variety of purposes, examples include identification of physical laws, the extrapolation of physical fields, pattern recognition, clustering, the approximation of multidimensional processes, forecasting without models, etc. In this study, the R package GMDH is presented to make short term forecasting through GMDH-type neural network algorithms. The GMDH package has options to use different transfer functions (sigmoid, radial basis, polynomial, and tangent functions) simultaneously or separately. Data on cancer death rate of Pennsylvania from 1930 to 2000 are used to illustrate the features of the GMDH package. The results based on ARIMA models and exponential smoothing methods are included for comparison.
\end{abstract}

\section{Introduction}

Time series data are ordered successive observations which are measured in equally or unequally spaced time. Time series data may include dependency among successive observations. Hence, the order of the data is important. Time series data appear in various areas and disciplines such as medical studies, economics, the energy industry, agriculture, meteorology, and so on. Modelling time series data utilizes the history of the data and makes forecasting using this history. At times, statistical models are not sufficient to solve some problems. Examples include pattern recognition, forecasting, identification, etc. Extracting the information from the measurements has advantages while modelling complex systems when there is not enough prior information and/or no theory is defined to model the complex systems. Selecting a model automatically is a powerful way for the researchers who are interested in the result and do not have sufficient statistical knowledge and sufficient time (Mueller et al., 1998) for an analysis.

The objective of this study is to develop an R package for forecasting of time series data. Some of recent softwares developed for time series are glarma, ftsa, MARSS, ensembleBMA, ProbForecastGOP, and forecast (Dunsmuir and Scott, 2015; Shang, 2013; Holmes et al., 2012; Fraley et al., 2011; Hyndman and Khandakar, 2008). In this study, we focused on the development of an R package for short term forecasting via Group Method of Data Handling (GMDH) algorithms. The history of GMDH-type neural network is based on works from the end of the 1960s and the beginning of the 1970s. First, Ivakhnenko (1966) introduced a polynomial, which is the basic algorithm of GMDH, to construct higher order polynomials. Also, Ivakhnenko (1970) introduced heuristic self-organization methods which constructed the main working system of GMDH algorithm. Heuristic self-organization method defines the way that the algorithm evolves, following rules such as external criteria. The GMDH method, convenient for complex and unstructured systems, has benefits over high order regression (Farlow, 1981).

Kondo (1998) proposed GMDH-type neural network in which the algorithm works according to the heuristic self-organization method. Kondo and Ueno $(2006 a, b)$ proposed a GMDH algorithm which has a feedback loop. According to this algorithm, the output obtained from the last layer is set as a new input variable, provided a threshold is not satisfied in the previous layer. The system of the algorithm is organized by a heuristic self-organization method where a sigmoid transfer function is integrated. Kondo and Ueno (2007) proposed a logistic GMDH-type neural network. The difference from a conventional GMDH algorithm was that the new one would take linear functions of all inputs at the last layer. Kondo and Ueno (2012) included three transfer functions (sigmoid, radial basis and polynomial functions) in the feedback GMDH algorithm. Srinivasan (2008) used a GMDH-type neural network and traditional time series models to forecast predicted energy demand. It was shown that a GMDH-type neural network was superior in forecasting energy demand compared to traditional time series models with respect to mean absolute percentage error (MAPE). In another study, Xu et al. (2012) applied a GMDH algorithm and ARIMA models to forecast the daily power load. According to their results, GMDH-based results were superior to the results of ARIMA models in terms of MAPE for forecasting performance.

There are some difficulties when applying a GMDH-type neural network. For example, there is no freely available software for researchers implementing the GMDH algorithms in the literature. We 
present the R package GMDH to make short term forecasting through GMDH-type neural network algorithms. The package includes two types of GMDH structures; namely, GMDH structure and revised GMDH (RGMDH) structure. Also, it includes a variety of options to use different transfer functions (sigmoid, radial basis, polynomial, and tangent functions) simultaneously or separately. Data on the cancer death rate of Pennsylvania from 1930 to 2000 are used to illustrate the implementation of GMDH package. We compare the results to those based on ARIMA models and exponential smoothing (ES) methods.

\section{Methodology}

In this section, data preparation, two types of GMDH-type neural network structures, and estimation of a regularization parameter in regularized least square estimation (RLSE) are given.

\section{Data preparation}

Data preparation has an important role in GMDH-type neural network algorithms. To get rid of very big numbers in calculations and to be able to use all transfer functions in the algorithm, it is necessary for range of the data to be in the interval of $(0,1)$. If $\alpha_{t}$ is the actual time series dataset at hand, this necessity is guaranteed by the following transformation,

$$
w_{t}=\frac{\alpha_{t}+\delta_{1}}{\delta_{2}}
$$

with

$$
\delta_{1}=\left\{\begin{array}{cc}
\left|\alpha_{t}\right|+1 & \text { if } \min \left(\alpha_{t}\right) \leq 0 \\
0 & \text { if } \min \left(\alpha_{t}\right)>0
\end{array}\right.
$$

and

$$
\delta_{2}=\max \left(\alpha_{t}+\delta_{1}\right)+1
$$

During the estimation and forecasting process in GMDH-type neural network algorithms, all calculations are done using the scaled data set, $w_{t}$. After all processes are ended-i.e, all predictions and forecasts are obtained-we apply the inverse transformation as follows,

$$
\widehat{\alpha}_{t}=\widehat{w}_{t} \times \delta_{2}-\delta_{1}
$$

Let's assume a time series dataset for $t$ time points, and $p$ inputs. An illustration of time series data structure in GMDH algorithms is presented in Table 1. Since we construct the model for the data with time lags, the number of observations, presented under the subject column in the table, is equal to $t-p$; and the number of inputs, lagged time series, is $p$. In this table, the variable called $z$ is put in the models as a response variable, and the rest of the variables are taken into models as lagged time series $x_{i}$, where $i=1,2, \ldots, p$. The notations in Table 1 are followed throughout this paper.

Table 1: An illustration of time series data structure in GMDH algorithms

\begin{tabular}{cccccc}
\hline Subject & $z$ & $x_{1}$ & $x_{2}$ & $\ldots$ & $x_{p}$ \\
\hline 1 & $w_{t}$ & $w_{t-1}$ & $w_{t-2}$ & $\ldots$ & $w_{t-p}$ \\
2 & $w_{t-1}$ & $w_{t-2}$ & $w_{t-3}$ & $\ldots$ & $w_{t-p-1}$ \\
3 & $w_{t-2}$ & $w_{t-3}$ & $w_{t-4}$ & $\ldots$ & $w_{t-p-2}$ \\
$\vdots$ & $\vdots$ & $\vdots$ & $\vdots$ & $\ddots$ & $\vdots$ \\
$t-p$ & $w_{p+1}$ & $w_{p}$ & $w_{p-1}$ & $\ldots$ & $w_{1}$ \\
\hline
\end{tabular}

A better model which explains the relation between response and lagged time series is captured via transfer functions. The sigmoid, radial basis, polynomial, and tangent functions, presented in Table 2, are mainly used to explain the relation between inputs and output in GMDH-type neural network algorithms (Kondo and Ueno, 2012). We use all transfer functions, stated in Table 2, simultaneously in each neuron. In other words, we construct four models at each neuron, and then the model which gives the smallest prediction mean square error (PMSE) is selected as the current transfer function at the corresponding neuron. 
Table 2: Transfer functions

\begin{tabular}{ll} 
Sigmoid Function & $z=1 /\left(1+e^{-y}\right)$ \\
Radial Basis Function & $z=e^{-y^{2}}$ \\
Polynomial Function & $z=y$ \\
Tangent Function & $z=\tan (y)$ \\
\hline
\end{tabular}

\section{GMDH algorithm}

GMDH-type neural network algorithms are modeling techniques which learn the relations among the variables. In the perspective of time series, the algorithm learns the relationship among the lags. After learning the relations, it automatically selects the way to follow in algorithm. First, GMDH was used by Ivakhnenko (1966) to construct a high order polynomial. The following equation is known as the Ivakhnenko polynomial given by

$$
y=a+\sum_{i=1}^{m} b_{i} \cdot x_{i}+\sum_{i=1}^{m} \sum_{j=1}^{m} c_{i j} \cdot x_{i} \cdot x_{j}+\sum_{i=1}^{m} \sum_{j=1}^{m} \sum_{k=1}^{m} d_{i j k} \cdot x_{i} \cdot x_{j} \cdot x_{k}+\ldots
$$

where $m$ is the number of variables and $a, b, c, d, \ldots$ are coeffients of variables in the polynomial, also named as weights. Here, $\mathrm{y}$ is a response variable, $x_{i}$ and $x_{j}$ are the lagged time series to be regressed. In general, the terms are used in calculation up to square terms as presented below,

$$
y=a+\sum_{i=1}^{m} b_{i} \cdot x_{i}+\sum_{i=1}^{m} \sum_{j=1}^{m} c_{i j} \cdot x_{i} \cdot x_{j}
$$

The GMDH algorithm considers all pairwise combinations of $p$ lagged time series. Therefore, each combination enters each neuron. Using these two inputs, a model is constructed to estimate the desired output. In other words, two input variables go in a neuron, one result goes out as an output. The structure of the model is specified by Ivakhnenko polynomial in equation 4 where $m=2$. This specification requires that six coefficients in each model are to be estimated.

The GMDH algorithm is a system of layers in which there exist neurons. The number of neurons in a layer is defined by the number of input variables. To illustrate, assume that the number of input variables is equal to $p$, since we include all pairwise combinations of input variables, the number of neurons is equal to $h=\left(\begin{array}{l}p \\ 2\end{array}\right)$. The architecture of GMDH algorithm is illustrated in Figure 1 when there are three layers and four inputs.

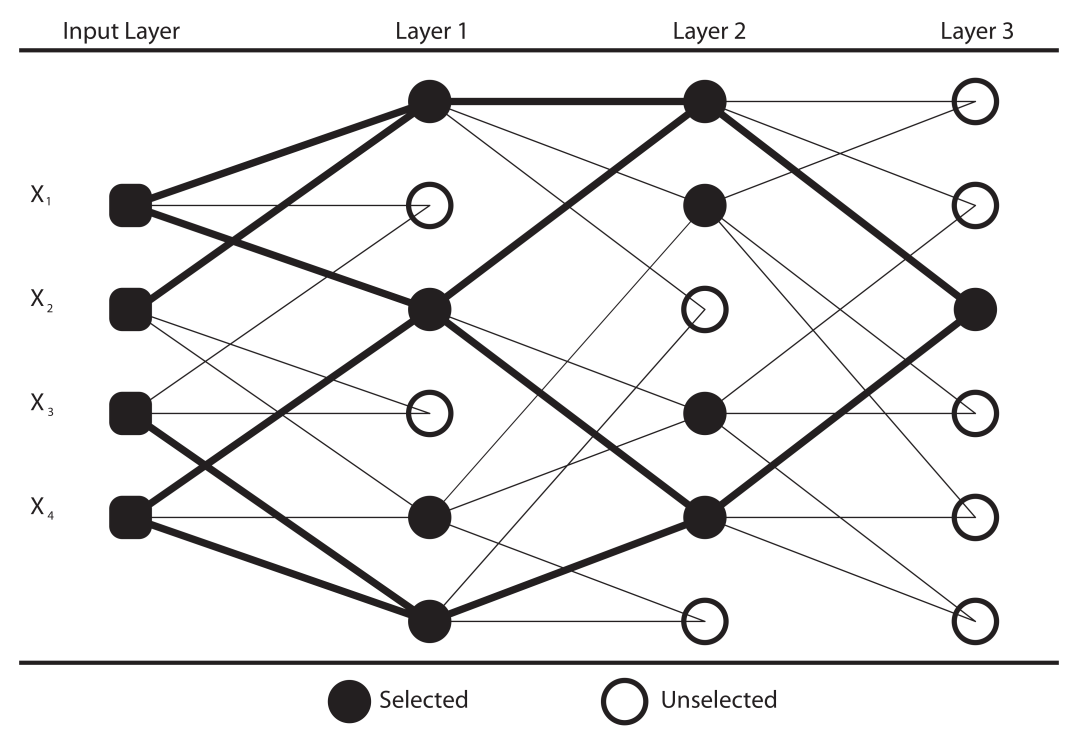

Figure 1: Architecture of GMDH algorithm

In the GMDH architecture shown in Figure 1, since the number of inputs is equal to four, the number of nodes in a layer is determined to be six. This is just a starting layer to the algorithm. The coefficients of equation 4 are estimated in each neuron. By using the estimated coefficients and input variables in each neuron, the desired output is predicted. According to a chosen external criteria, $p$ 
neurons are selected and $h-p$ neurons are eliminated from the network. In this study, prediction mean square error (PMSE) is used as the external criteria. In Figure 1, four neurons are selected while two neurons are eliminated from the network. The outputs obtained from selected neurons become the inputs for the next layer. This process continues until the last layer. At the last layer, only one neuron is selected. The obtained output from the last layer is the predicted value for the time series at hand. The flowchart of the algorithm is depicted in Figure 2.

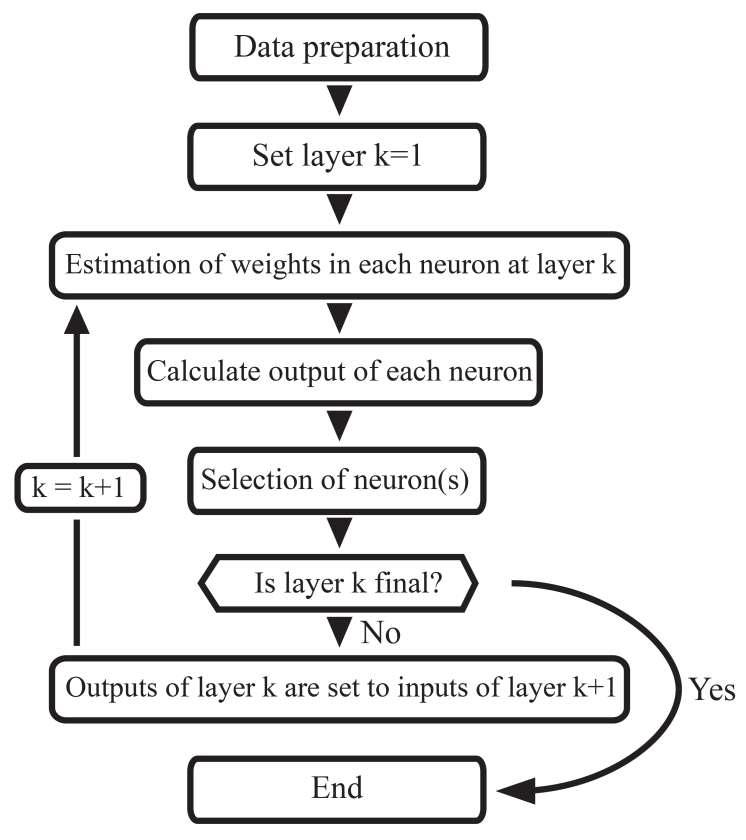

Figure 2: Flowchart of GMDH algorithms

In a GMDH algorithm, there exist six coefficients to be estimated in each model. Coefficients are estimated via RLSE.

\section{RGMDH algorithm}

A GMDH-type neural network constructs the algorithm by investigating the relation between two inputs and the desired output. Architecture of a revised GMDH (RGMDH)-type neural network does not only consider this relation, but it also considers the individual effects on the desired output (Kondo and Ueno, 2006b). There are two different types of neurons in an RGMDH-type neural network. In the first type of neuron, it is same as in GMDH-type neural network, given as in equation 4. That is, two inputs enter the neuron, one output goes out. In the second type of neuron, $r$ inputs enter the neuron, one output goes out. This second type neuron is given by

$$
y=a+\sum_{i=1}^{r} b_{i} \cdot x_{i} \quad, \quad r \leq p,
$$

where $r$ is the number of inputs in the corresponding second type neuron.

As mentioned above, there exist $h=\left(\begin{array}{l}p \\ 2\end{array}\right)$ neurons in one layer in a GMDH-type neural network. In addition to this, with the $p$ neurons from the second type of neuron, the number of neurons in one layer becomes $\eta=\left(\begin{array}{l}p \\ 2\end{array}\right)+p$ in an RGMDH-type algorithm. The architecture of an RGMDH-type algorithm is shown in Figure 3 for the case when there are three layers and three inputs. In this architecture, since the number of inputs is three, the number of nodes in a layer is determined to be six. Here, three of six nodes are the first type of neurons in which all pairwise combinations of lagged time series are already used as in the GMDH algorithm. The rest of the three nodes are the second type of neurons where the individual effects of the lagged time series are sequentially added to the layer starting from lag 1 . In each neuron, coefficients of models are calculated by using the corresponding models in equations 4 and 5. For instance, in Figure 3, there are six coefficients to be estimated as given by equation 4 for the first type of neurons, and two, three and four coefficients are estimated as given in equation 5 for the the second type of neurons when $r$ equals to 1, 2 and 3, respectively. The desired output is predicted by utilizing estimated coefficients and input variables in each neuron. Here, $p$ neurons are selected as living cells and $\eta-p$ death cells are eliminated from the network according to the external criteria. The rest of the algorithm is same with GMDH. 


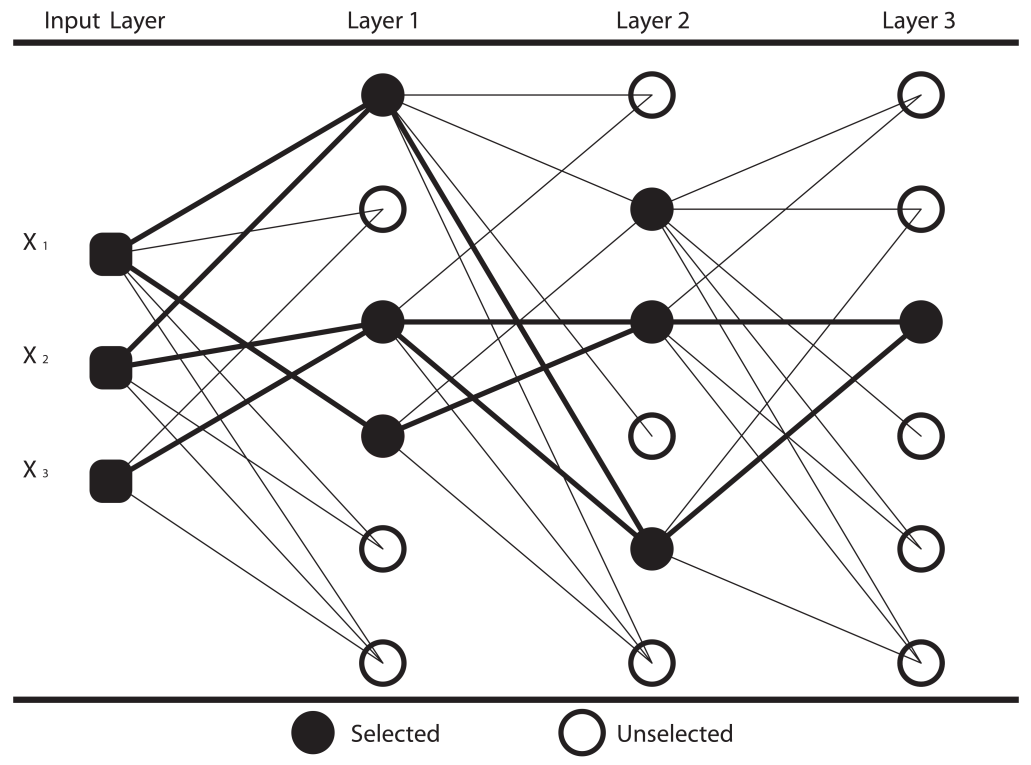

Figure 3: Architecture of an RGMDH algorithm

\section{Estimation of regularization parameter in RLSE}

In each estimation step, there exist the coefficients to be estimated. While we are estimating these coefficients, we use the regularized least square estimation method. It is stated that regularized least square estimation is utilized when there is the possibility of a multi-collinearity problem by integrating a regularization parameter, $\lambda$, into the estimation step. It is important to note that regularized least square estimation differs from the least square estimation when the regularization parameter is not zero.

We integrate the estimation of a regularization parameter (penalizing term) via validation in GMDH algorithms. For this purpose, we divide the data into two parts: a learning set and a testing set. In the GMDH package, $70 \%$ of the time series, by default, is taken for the learning set. Since the data set is time dependent, the order of data is saved in this division process. In other words, by default, the first $70 \%$ of the data is used for learning set and the last $30 \%$ of the data is utilized as a testing set. This whole process is applied for each model constructed in each neuron. The algorithm for the regularization parameter estimation is as follows:

i) Clarify the possible regularization parameter, $\lambda=0,0.01,0.02,0.04,0.08, \ldots, 10.24$. Note that, when $\lambda=0$, RLSE is converted to LSE.

ii) For each possible $\lambda$ value, coefficients are estimated via RLSE by using the learning set.

iii) After the calculation of coefficients, calculate the predicted values by utilizing the test set to obtain the MSE for each regularization parameter.

iv) Select the regularization parameter which gives the minimum MSE value.

\section{Implementation of GMDH package}

The data used in this application of the GMDH package are the yearly cancer death rate (per 100,000 population) in the Pennsylvania between 1930 and 2000. The data were documented in Pennsylvania Vital Statistics Annual Report by the Pennsylvania Department of Health in 2000 (Wei, 2006). This dataset is also available as a dataset in the package GMDH. After installing the GMDH package, it can be loaded into an $\mathrm{R}$ workspace by

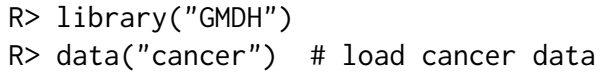

After the cancer death rate data set is loaded, one may use fcast function in GMDH package for short-term forecasting. To utilize the GMDH structure for forecasting, method is set to "GMDH". One should set the method to "RGMDH" to use the RGMDH structure. 
$R>$ out $=f$ cast $($ cancer $[1: 66]$, method $=$ "GMDH", input $=15$, layer $=1, f$. number $=5$, level $=95, \mathrm{tf}=$ "all", weight $=0.70$, lambda $=\mathrm{c}(0,0.01,0.02,0.04,0.08,0.16$, $0.32,0.64,1.28,2.56,5.12,10.24)$ )

$\begin{array}{lrrrr} & \text { Point } & \text { Forecast } & \text { Lo 95 } & \text { Hi } 95 \\ 67 & 249.5317 & 244.9798 & 254.0836 \\ 68 & & 249.6316 & 244.4891 & 254.7741 \\ 69 & & 248.9278 & 243.0318 & 254.8239 \\ 70 & & 247.0385 & 240.7038 & 253.3731 \\ 71 & & 244.7211 & 237.1255 & 252.3168\end{array}$

\# display fitted values

R> out\$fitted

\# return residuals

R> out\$residuals

\# show forecasts

R> out\$mean

In this part, we divided the data into two parts for the aim of observing the ability of methods on prediction $(n=66)$ and forecasting $(n=5)$. We include ARIMA models and ES methods for comparison purpose. For the determination of the best order of ARIMA models and the best method of ES techniques, there are two functions in the R package forecast. These functions, auto.arima and ets, which use grid search, select the best model according to the criteria of either AIC, AICc or BIC. For this data set, the functions suggested the model ARIMA $(1,1,0)$ with intercept and an ES method with multiplicative errors, additive damped trend and no seasonality $(\mathrm{M}, \mathrm{Ad}, \mathrm{N})$, respectively. We also added the model ARIMA $(0,1,0)$ with intercept for this data set suggested by Wei (2006). For all models, prediction mean square error (PMSE) and forecasting mean square error (FMSE) are stated in Table 3.

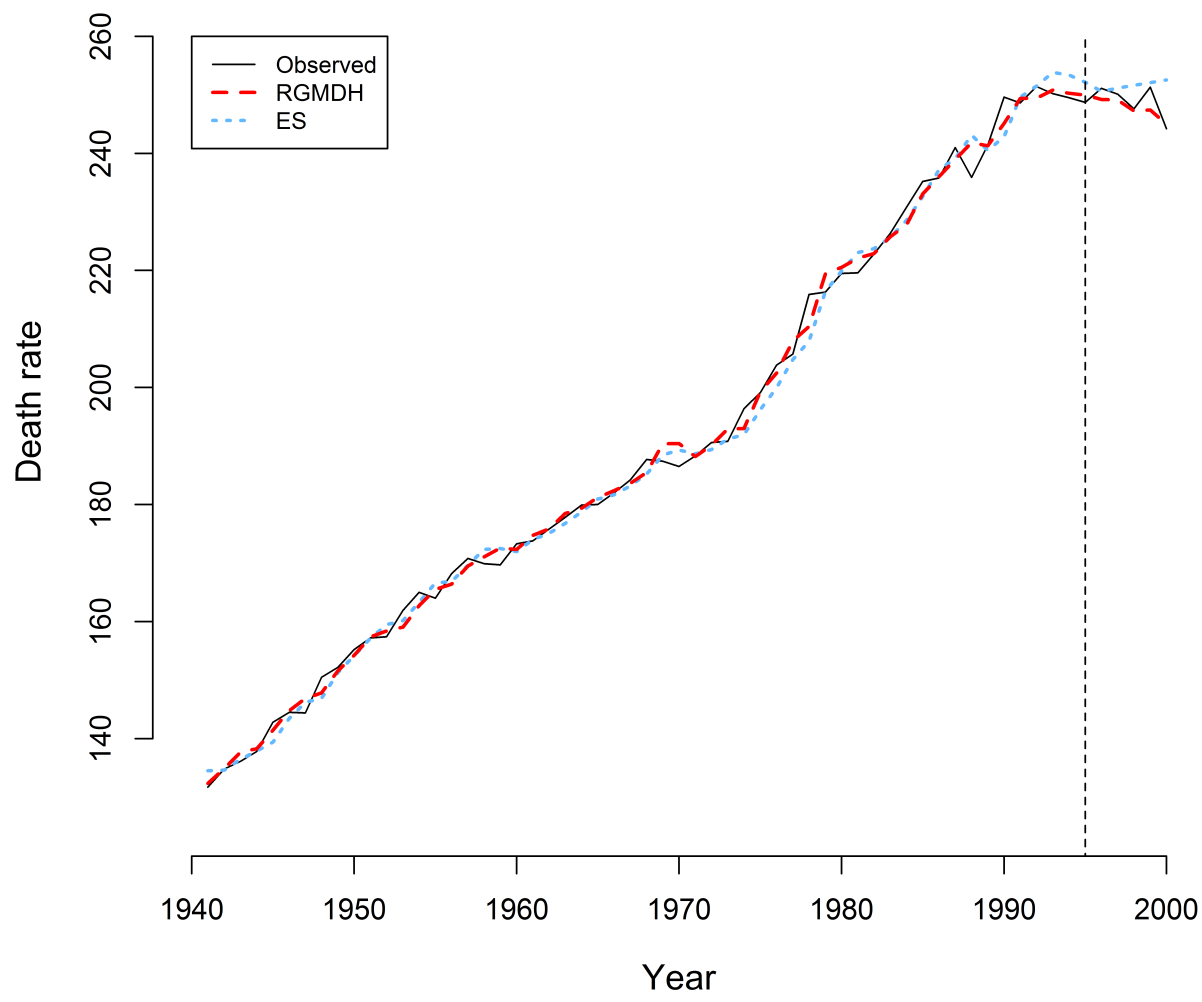

Figure 4: Yearly cancer death rate (per 100,000 population) in Pennsylvania between 1941 and 2000 with predictions and forecasts obtained via RGMDH and ES(M,Ad,N) 
Table 3: Comparison of the GMDH algorithms with other models on cancer death rate in terms of prediction mean square error (PMSE) and forecasting mean square error (FMSE)

\begin{tabular}{lrr}
\hline & PMSE & FMSE \\
\hline GMDH & 4.985 & 4.575 \\
RGMDH & 4.287 & 4.102 \\
ARIMA $(1,1,0)$ with intercept & 5.995 & 81.874 \\
ARIMA(0, 1, 0) with intercept & 6.324 & 73.756 \\
ES (M, Ad, N) & 6.153 & 17.508 \\
\hline
\end{tabular}

The best forecasting performance belongs to the RGMDH algorithm and its prediction accuracy also yields better results as compared to the GMDH, ARIMA and ES models. Moreover, the GMDH algorithm outperforms the ARIMA and ES models in prediction and forecasting. To avoid visual pollution in Figure 4, we include only the predictions and forecasts of RGMDH algorithm and ES (M, Ad, N).

\section{Conclusion}

In this study, we used GMDH-type neural network algorithms, the heuristic self-organization method for the modelling of complex systems, to make forecasts for time series data sets. Our primary focus was to develop a free software implementation. Concretely, we developed an R package GMDH to make forecasting in the short term via GMDH-type neural network algorithms. Also, we included different transfer functions (sigmoid, radial basis, polynomial, and tangent functions) into the GMDH package. Our R package allows that these functions can be used simultaneously or separately, as desired.

In the estimation of coefficients, since we construct the model for the data with lags, there exists a high possibility of there occurring a multi-collinearity problem. Therefore, we utilized regularized least square estimation to handle such occurences. It is important to note that estimation of a regularization parameter is the question of interest. Validation was applied in order to estimate the regularization term. After selection of a regularization term, coefficients were estimated by the help of all observations and the regularization parameter.

Application of the algorithms on a real life dataset suggests improved performance of GMDH-type neural network algorithms over ARIMA and ES models in prediction and short term forecasting. Researchers are able to use GMDH algorithms easily since our R package GMDH is available on Comprehensive R Archive Network (CRAN) at http: / /CRAN . R-project . org / package=GMDH.

Future studies are planned in the direction of transfer functions. In this study, we used four different transfer functions - sigmoid, radial basis, polynomial, and tangent functions - into GMDH algorithms. We plan to integrate the Box-Cox transformation into GMDH algorithms. GMDH algorithms with four transfer functions and GMDH algorithms with Box-Cox transformation are going to be performed on real data applications to compare the prediction and short term forecasting. After being documented, the related GMDH algorithms with the Box-Cox transformation are going to be implemented in the R package GMDH.

\section{Acknowledgment}

We thank the anonymous reviewers for their constructive comments and suggestions which helped us to improve the quality of our paper.

\section{Bibliography}

W. T. M. Dunsmuir and D. J. Scott. The glarma package for observation-driven time series regression of counts. Journal of Statistical Software, 67(7):1-36, 2015. doi: 10.18637/jss.v067.i07. [p379]

S. J. Farlow. The GMDH algorithm of Ivakhnenko. The American Statistician, 35(4):210-215, 1981. [p379]

C. Fraley, A. E. Raftery, T. Gneiting, J. Sloughter, and V. J. Berrocal. Probabilistic weather forecasting in R. The R Journal, 3(1):55-63, 2011. [p379]

E. E. Holmes, E. J. Ward, and K. Wills. MARSS: Multivariate autoregressive state-space models for analyzing time-series data. The R Journal, 4(1):30, 2012. [p379] 
R. Hyndman and Y. Khandakar. Automatic time series forecasting: The forecast package for R. Journal of Statistical Software, 27(3):1-22, 2008. [p379]

A. Ivakhnenko. The group method of data handling-a rival of the method of stochastic approximation. Soviet Automatic Control, 13(3):43-55, 1966. [p379, 381]

A. Ivakhnenko. Heuristic self-organization in problems of engineering cybernetics. Automatica, 6(2): 207-219, 1970. [p379]

T. Kondo. GMDH neural network algorithm using the heuristic self-organization method and its application to the pattern identification problem. In SICE'98. Proceedings of the 37th SICE Annual Conference. International Session Papers, pages 1143-1148. IEEE, 1998. [p379]

T. Kondo and J. Ueno. Medical image recognition of the brain by revised GMDH-type neural network algorithm with a feedback loop. International Journal of Innovative Computing, Information and Control, 2(5):1039-1052, 2006a. [p379]

T. Kondo and J. Ueno. Revised gmdh-type neural network algorithm with a feedback loop identifying sigmoid function neural network. International Journal of Innovative Computing, Information and Control, 2(5):985-996, 2006b. [p379, 382]

T. Kondo and J. Ueno. Logistic GMDH-type neural network and its application to identification of X-ray film characteristic curve. JACIII, 11(3):312-318, 2007. [p379]

T. Kondo and J. Ueno. Feedback GMDH-type neural network and its application to medical image analysis of liver cancer. In 42th ISCIE international symposium on stochastic systems theory and its applications, pages 81-82, 2012. [p379, 380]

J. A. Mueller, A. Ivachnenko, and F. Lemke. GMDH algorithms for complex systems modelling. Mathematical and Computer Modelling of Dynamical Systems, 4(4):275-316, 1998. [p379]

H. L. Shang. ftsa: An R package for analyzing functional time series. The R Journal, 5(1):64-72, 2013. URL http://journal.r-project.org/archive/2013-1/shang.pdf. [p379]

D. Srinivasan. Energy demand prediction using GMDH networks. Neurocomputing, 72(1):625-629, 2008. [p379]

W. W. S. Wei. Time series analysis: univariate and multivariate methods. Addison-Wesley publ, 2006. [p383, 384]

H. Xu, Y. Dong, J. Wu, and W. Zhao. Application of GMDH to short-term load forecasting. In Advances in Intelligent Systems, pages 27-32. Springer-Verlag, 2012. [p379]

\author{
Osman Dag \\ Department of Biostatistics \\ Faculty of Medicine \\ Hacettepe University \\ 06100 Ankara, Turkey \\ osman. dag@hacettepe. edu. tr \\ Ceylan Yozgatligil \\ Department of Statistics \\ Faculty of Arts and Sciences \\ Middle East Technical University \\ 06531 Ankara, Turkey \\ ceylan@metu. edu. tr
}

